\title{
La nutrition azotée de l'Épicéa en sol engorgé : étude expérimentale
}

\author{
G. LÉVY \\ avec la collaboration technique de Maris-Louise Deeu, C. Brechet el Y. LfFevre \\ Station de Recherches sur les Sols forestiers et la Fertilisation \\ Centre de Recherches forestieres de Nancy, I.N.R.A., \\ Champenoux, 54280 Seichamps
}

\begin{abstract}
Résumé
Dans les conditions expérimentales, de jeunes plants d'Epicéa ne commençaient à assimiler Vazote que lorsque la nappe atteignait au minimum $6 \mathrm{~cm}$ de profondeur environ. Ensuite, les teneurs foliaires en azote élaient d'autant meilleures que la nappe était plus profonde.

11 a été établi que la nutrition azotée de l'Epicéa en sol engorgé dépend de la disponibilité d'azote nitrique. D'une part. en effet, l'azote apporté sous forme de $\mathrm{NH}_{4}$ ' n'a pas du tout été assimilé en présence d'une nappe, même profonde, contrairement à la forme $\mathrm{NO}_{3}{ }^{-}$. D'autre part, la tencur en air minimum permettant la nitrification a été atteinte, à proximité de la surface du sol, lorsque la nappe affleurait à $-6 \mathrm{~cm}$ : l'assimilation de l'azote par les plants débutait pour ce même niveau.

Des essais en inrubation ont lait apparaître que différents phénomènes pouvaient contribuer, en forêt, à prolonger la période de carence azotée de l'Epicéa. Ainsi, chaque fois que la nappe affleurait à proximité de la surface du sol, la nitrification ne reprend qu'après une assez longue période de ressuyage; de plus, il se produit alors une perte d'azote minéral. Si l'engorgement a été de longue durée, il y a formation, lors de la reprise de la nitrification, d'une grande quantité de $\mathrm{NO}_{;}$pendant une courte période de temps: des pertes de nitrates peuvent donc intervenir par lessivage.
\end{abstract}

\section{I. - Introduction}

Des études précédentes en milieu contrôlé (L.EVY, 1981) ont permis de montrer que de jeunes plants d'Epicéa commun (Picea abies) ne prélevaient sans doute plus d'azote dans le sol dès l'installation d'une nappe superficielle, même si celle-ci est renouvelée très fréquemment. Cette sévère carence azotée contribue, dans les cas défavorables, à accélérer l'apparition et la progression de la mortalité des plants, et, toujours, à freiner leur croissance.

Il est normal que les conditions hypoxiques créées par la nappe au niveau racinaire affectent le prélèvement par les plantes des éléments minéraux dans le 
sol (Heller, 1969) ; cette carence azotée est cependant étonnante en raison de son importance et de la rapidité de son apparition; ainsi, dans le cas de jeunes plants d’Epicéa, le déficit en phosphore et potassium n’apparaît que bien plus tardivement.

Aussi avons-nous tenté de comprendre les raisons de cette soudaine carence en azote, qui apparaît également en forêt, sur sols à forte hydromorphie temporaire, aussi bien sur jeunes plants que sur arbres adultes (LEvy, 1978).

Nous n'avons pas étudié l'absorption proprement dite de l'azote, mais uniquement les teneurs des aiguilles en cet élément : nous utiliserons donc le terme d' $\mathrm{d}$ assimilation» de préférence à celui d" «absorption ».

Nous examinerons d'abord dans quelle mesure la carence azotée apparaît encore dans le cas de nappes non superficielles. Nous traiterons ensuite de l'influence de différents niveaux d'engorgement sur l'assimilabilité des diverses formes d'azote, puis sur la disponibilité dans le sol des formes assimilables. Enfin, nous tenterons d'expliquer les résultats concernant la nutrition azotée de l'Epicéa en forêt par la disponibilité de l'azote assimilable au cours d'une saison de végétation.

\section{II. - Matériel et méthodes}

L'étude de l'assimilation de l'azote par l'Epicéa a été effectuée en serre, sur des plants âgés de 2 ans, dans des vases de végétation cylindriques d'environ $30 \mathrm{~cm}$ de diamètre et de hauteur. Le fond de ces vases est garni d'une hauteur de $5 \mathrm{~cm}$ de gravillons, au-dessus desquels se trouve le sol, dont la composition sera précisée lorsque nous traiterons de chacune des expériences. Nous avons utilisé un horizon $A_{1}$ qui est un mull mésotrophe à texture limoneuse, dont le rapport $\mathrm{C} / \mathrm{N}$ est de 14 , le $\mathrm{pH}$ à l'eau de 5,1 et le taux de matière organique de $5 \%$; il a été prélevé dans un sol à pseudogley. La description détaillée des vases de végétation, notamment du dispositif destiné à établir le niveau de la nappe à la hauteur désirée, a été faite précédemment (LEVY, 1981). Il s’agit d'expériences en blocs (en général, 5 répétitions, 9 individus par vase).

Les plants ont été engorgés au cours de l'année même de leur repiquage, après le temps néccssaire à leur installation, et alors que la nouvelle pousse terminale atteignait en moyenne $1 \mathrm{~cm}$ de long.

Lanalyse de lazote total (pourcentage et quantité totale dans les aiguilles de l'année) al été effectuée périodiquement sur des plants représentatifs du traitement considéré.

Afin d'étudier les problèmes d'absorption de l'azote sous forme $\mathrm{NH}_{4}{ }^{+}$ou $\mathrm{NO}_{3}^{-}$, nous avons utilisé un substrat dépourvu de matière organique (paragraphe 32 ) ; nous avons alors apporté dans tous les vases, 10 jours avant l'engorgement, $50 \mathrm{ml}$ de solution de phosphate monopotassique à $40 \mathrm{~g} / \mathrm{l}$. Nous avons ensuite «lavé»le sol juste avant l'engorgement. Au cours de celui-ci, nous avons effectué tous les 15 jours dans chaque vase des apports, soit de $\mathrm{NO}_{3}-50 \mathrm{ml}$ de solution de nitrate de calcium à $25 \mathrm{~g} / \mathrm{l}$, soit de $\mathrm{NH}_{4}+: 50 \mathrm{ml}$ d'acétate d'ammonium à $17 \mathrm{~g} / \mathrm{l}$, puis nous avons "lavé » le sol quelques jours après chacun de ces apports. 
Des expériences en incubation ont été réalisées dans une étuve matintenue à $27{ }^{\circ} \mathrm{C}$, l'évaporation étant selon le cas favoriséc ou empêchée. Nous avons utilisé de petits récipients cylindriques de $9 \mathrm{~cm}$ de hauteur et $7 \mathrm{~cm}$ de diamètre contenant un petit piézomètre, percé sur toute sa hauteur, permettant de mettre ou de retirer leau de la nappe. Ces récipients sont remplis avec l'horizon $A_{1}$ déjà utilisé dans les vases de végétation. $\mathrm{Ce}$ sol est soumis selon le cas à différentes conditions hydriques : nappe en surface ou à $-4 \mathrm{~cm}$, maintien à la capacité au champ, déssèchement.

Des analyses des teneurs en $\mathrm{NH}_{4}{ }^{+}, \mathrm{NO}_{3}{ }^{-}$, NO.-- ont été effectuées périodiquement sur le sol; les quantités contenues dans l'eau provenant du ressuyage ont été, lorsqu'il le fallait, ajoutées aux valeurs trouvées dans le sol. I.es teneurs en azote nitreux s'étant avérées toujours négligeables, nous ne parlerons plus de cette forme d'azote minéral.

\section{III. - Résultats et commentaires}

\section{1. - Influence de la profondeur de la nappe sur la tencur foliaire en azote}

Nous avions déjà observé au cours d'une expérienec précédente (LEVY, 1971) qu'une nappe maintenue à $-14 \mathrm{~cm}$ et surtout à $-7 \mathrm{~cm}$ provoquait un déficit foliaire en azote sur de jeunes plants d'Epicéa repiqués sur un substrat formé d'un horizon $A_{1}$ limoneux recouvrant un horizon $A_{2}$ de même texture ; après 45 jours d'engorgement, on notait les valeurs suivantes : sol à la capacité au champ : 2,15 p. 100, nappe à $-14 \mathrm{~cm}: 1,71$ p. 100 , nappe à $-7 \mathrm{~cm}: 1,03$ p. $100 \mathrm{de} \mathrm{N}$.

Nous avons étendu cette étude à d'autres substrats, en effectuant les analyses foliaires après des durées variées d'engorgement. Les résultats sont schématisés sur la figure 1, pour des durées d'engorgement de 27 et 82 jours $(1 \mathrm{a}), 39$ et 76 jours (1 b). 1 a correspond à un substrat formé uniquement d'horizon $\Lambda_{1}$, et 1 b à $8 \mathrm{~cm}$ d'horizon $A_{1}$ surmontant un sable.

L'analyse a été faite sur des plants moyens pour le traitement considéré (et en bon état physiologique apparent), sauf pour la nappe à - $6 \mathrm{~cm}$, lors du prélèvement à 82 ou 76 jours : dans chacune des deux expériences, les plants correspondants se divisaient alors nettement en deux lots, les uns bien verts (ceux dont la teneur en azote est la plus élevée), les autres de teinte vert-jaunc.

La figure 1 montre une liaison très nette entre la profondeur d'affleurement de la nappe et la teneur des aiguilles en azote; l'absorption deviendrait généralement de plus en plus faible au fur et à mesure de la remontée de la nappe vers la surface. Il semblerait même qu'il existe un certain seuil d'absorption correspondant à unc nappe assez voisine de $-6 \mathrm{~cm}$.

Quand la nappe est située à $-6 \mathrm{~cm}$, il apparaît en effet, au moins après unc certaine durée d'engorgement (phénomène constaté lors de la $2^{\mathrm{c}}$ date), une assimilation dazote se rapprochant du «tout ou rien». En fait, il n'est pas impossible que les plants qui assimilent alors l'azote soient très légèrement surélevés, de $1 \mathrm{~cm}$ au maximum, par rapport aux autres (pour eux, la nappe est donc voisine de 
a

1er substrot ( $A_{1}$ pur)

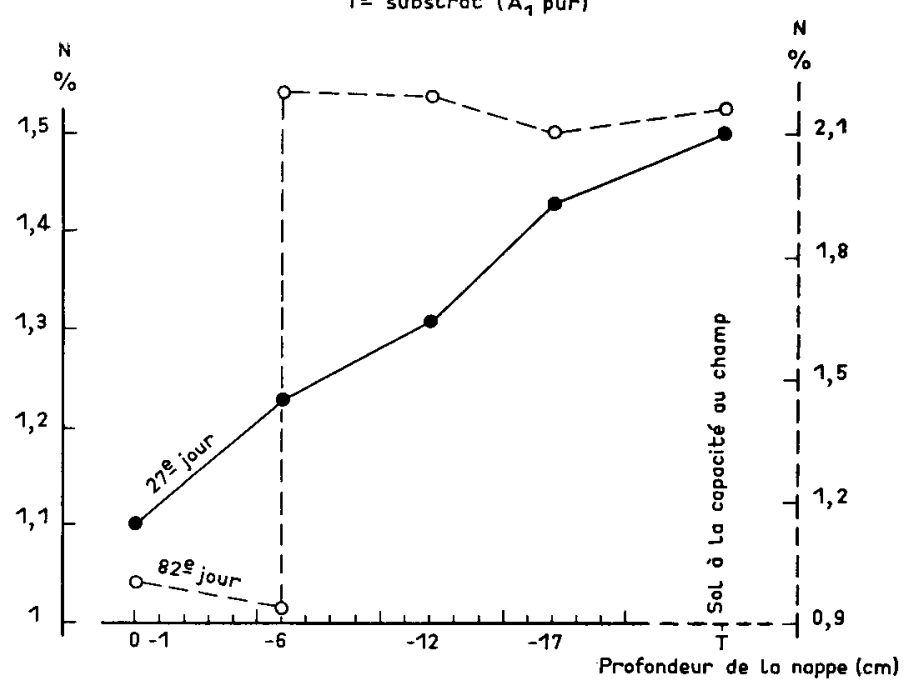

b

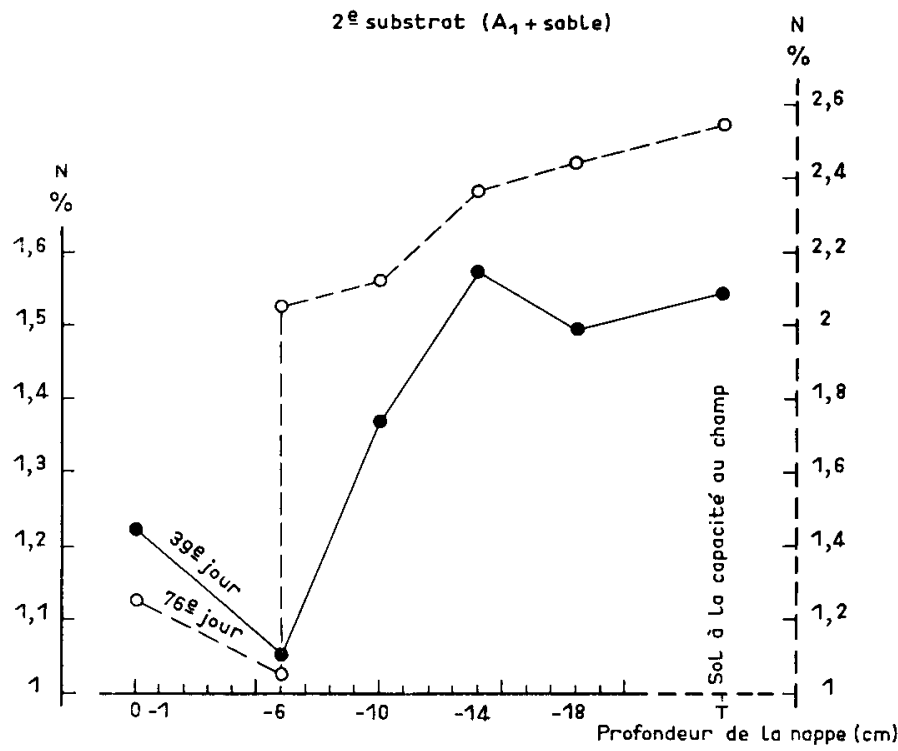

FIG. 1

Influence de la profondeur d'affleurement de la nappe sur la concentration foliaire en azote Influence of water-table depth on nitrogen foliar content 
- $7 \mathrm{~cm}$ ) ; on constate d'ailleurs qu'ils ont, à la fin de l'engorgement, un système racinaire nettement plus développé que les autres. Le fait que la teneur en azote soit plus élevée quand la nappe est en surface que lorsqu'clle est maintenue à $-6 \mathrm{~cm}$ est sans doute dû à un phénomène de dilution. L'échantillon $-6 \mathrm{~cm}$ au $27^{\prime \prime}$ jour sur la figure 1 a est probablement constitué surtout de plants qui seraient devenus bien verts par la suite.

Dans l'expérience correspondant à la figure $1 \mathrm{a}$, le maximum de concentration foliaire semble, au $82^{\circ}$ jour, être atteint dès que la nappe permet une assimilation d'azote $(-6$ ou $-7 \mathrm{~cm})$.

Par contre, dans celle de la figure $1 \mathrm{~b}$, la teneur en $\mathrm{N}$ à 76 jours, bien quassez élevée, reste encore inférieure au maximum lorsque la nappe est à $-6 \mathrm{~cm}$, et s'cn rapproche progressivement lorsque la nappe s'abaisse. La profondeur de la nappe semble donc agir alors de manière un peu différente dans les deux expériences. Cela peut provenir de ce que le substrat correspondant à la figure 1 a est nettement plus favorable à la croissance des plants que celui de la figure 1 b, du moins s'il n'est pas trop engorgé ; les différences de longueur de pousse sont beaucoup plus marquées selon la profondeur de nappe en 1 a qu'en $1 \mathrm{~b}$ : il y aurait alors un phénomène de dilution de l'azote; de plus, en 1 a, des pousses "d'août», un peu plus pauvres en azote que les pousses normales, sont apparues dans les traitements $-17 \mathrm{~cm}$ et $\mathrm{T}$, abaissant ainsi la teneur moyenne. La courbe obtenue en 1 b serait alors celle qui correspond le mieux aux possibilités réelles d'absorption de l'azote.

Une certaine limite, relative ou absolue, d'assimilation de lazote par les jeunes plants d'Epicća correspond done à une nappe située non loin de $-6 \mathrm{~cm}$; au-delà, la concentration foliaire en azote est fonction de la profondeur de la nappe. Comment peut-on expliquer ces résultats? On peut supposer que la réponse fait intervenir la forme de l'azote. En effet, on sait qu'en sol sain les jeunes plants d'Epicéa peuvent absorber à la fois la forme $\mathrm{NH}_{4}^{-+}$et la forme $\mathrm{NO}_{3}^{-}$. Mais on sait également :

- que labsorption de $\mathrm{NH}_{4}+$ exige chez la plupart des végétaux des conditions d'oxygénation supéricures à celles de $\mathrm{NO}_{3}^{-}$(Heller, 1969),

- qu'en milieu engorgé, si l'ammonification est en principe aussi active qu’en sol sain, il n'y a en revanche pas de nitrification (DOMmergues et MANGENOT, 1970).

Mais qu'en est-il de ces deux phénomènes dans le cas qui nous intéresse. c'est-à-dire nappe située de 0 à -14 ou $-17 \mathrm{~cm}$ ? Les expériences décrites ci-dessous sont destinées à vérifier que les déficits en azote relatés précédemment (jeunes plants d'Epicéa) sont dus au fait que $\mathrm{NO}_{3^{-}}^{-}$est la seule forme assimilable dans les conditions expérimentales, mais que cette forme est alors soit absente (nappe située entre la surface et $-6 \mathrm{~cm}$ ), soit en quantité insuffisante (nappe plus profonde).

\section{2. - Assimilation des formes $\mathrm{NH}_{4}{ }^{+}$et $\mathrm{NO}_{3}^{-}$an fonction de la profonder de la nappe}

Nous avons apporté à plusieurs reprises de l'azote sous forme soit $\mathrm{NH}_{4}{ }^{+-}$, soit $\mathrm{NO}_{3}:-$ (voir «méthodes ») à de jeuncs plants repiqués sur substrat dépourvu de matière organique (même texture que l'horizon $A_{1}$ utilisé précédemment : c'est l'horizon $A_{2}$ du même sol brun lessivé à pseudogley), dans des vases à niveaux d'engorgement 
variés. L'expérience 1 de la figure 2 représente les teneurs correspondantes de l'azote dans les aiguilles après environ 1,5 mois d'engorgement.

Des dosages d'azote ont été effectués également après des durées d'engorgement différentes; ils ont conduit à des courbes de même allure, mais avec souvent une différence moins marquée (voire nulle) entre l'effet d'un apport de $\mathrm{NO}_{3}^{-}$et celui d'un apport de $\mathrm{NH}_{4}+$ dans le cas de plants non engorgés.

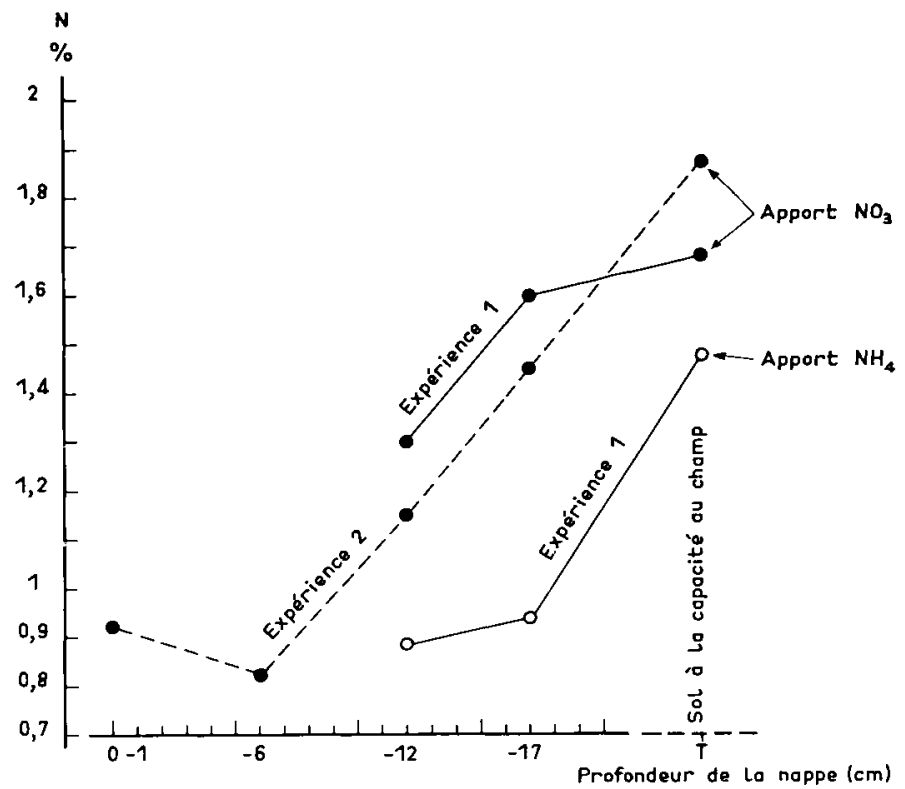

FIG. 2

Influence de la forme sous laquelle l'azote est apporté sur la concentration foliaire en cet élément

Influence of the form in which nitrogen is brought $\left(\mathrm{NH}_{4}+\right.$ or $\left.\mathrm{NO}_{3}-\right)$ on nitrogen foliar content

On constate sur la figure 2 que, dans le cas d'apports de $\mathrm{NO}_{3}{ }^{-}$, la teneur foliaire en $\mathrm{N}$ pour une nappe située à $-17 \mathrm{~cm}$ est presque aussi élevée que pour le témoin non engorgé, alors qu'elle est nettement plus faible pour une nappe maintenue à $-12 \mathrm{~cm}$. Par contre, dans le cas d'apports de $\mathrm{NH}_{4}{ }^{+}$, le taux d'azote foliaire est toujours très faible en présence d'une nappe, qu'elle soit situéc à -12 ou $-17 \mathrm{~cm}$; il semble même qu'alors l'azote ne soit pas du tout assimilé, car dans ces deux situations, la teneur totale en azote (en $\mathrm{mg}$ ) de l'ensemble des aiguilles de l'année d'un plant n’augmente pas avec le temps; il s'agit d'un résultat moyen, car la biomasse foliaire varie assez sensiblement d'un plant à l'autre : or les analyses successives concernent évidemment des plants différents. 
L'expérience 2 (sur même substrat) représentée sur la figure 2 , bien qu'en fait non conçue dans le même but, a été réalisée dans des conditions comparables; elle permet de confirmer que $\mathrm{NO}_{3}-$ est assimilé lorsque la nappe est situćc à $-17 \mathrm{~cm}$, mais, ici, moins bien que pour le témoin non engorgé, peut-être parce que les apports de $\mathrm{NO}_{3}^{-}$étaient moins importants que dans l'expérience $1 ; \mathrm{NO}_{3}{ }^{-}$est aussi assimilé partiellement lorsque la nappe affleure à $-12 \mathrm{~cm}$. On peut penser que, dans ce cas, la limite d'assimilation de $\mathrm{NO}_{3}-$ correspond à une nappe voisine $\mathrm{de}-7 \mathrm{ou}-8 \mathrm{~cm}$.

La seule forme d'azote assimilable en présence d'une nappe, même relativement assez profonde $(-17 \mathrm{~cm})$, est done $\mathrm{NO}_{3}-$. On peut d'ailleurs remarquer un certain parallélisme entre les concentrations d'azote en fonction de la profondeur représentées d'une part sur la figure 1, d'autre part sur la figure 2 (dans le cas d'un apport de $\mathrm{NO}_{3}{ }^{-}$) : cela tend à montrer qu'en sol naturel engorgé, c'est done bien à partir de la forme $\mathrm{NO}_{3}-$ que l'azote est assimilé ; mais dans quelles conditions $\mathrm{NO}_{3}-$ est-il alors disponible?

\section{3. - Apparition de la nitrification en présence d'une nappe}

S'il n'y a en général pas du tout de nitrification lorsque l'horizon organique d'un sol est engorgé (Dommergues \& Mangenot, 1970), qu'en est-il si la nappe n'affleure pas en surface, mais est à l'origine de fortes remontées capillaires ?

Nous avons constaté, en opérant par incubation de l'horizon $A_{1}$ utilisé dans nos expériences, que la nitrification, évidemment nulle dans le cas d'une nappe maintenue en surface, est également totalement absente lorsque la nappe est à $-4 \mathrm{~cm}$; on pourra le constater sur la figure 4 (voir paragraphe 3.4.).

Nous n'avons pas, pour des raisons matérielles, effectué la même opération avec des nappes plus profondes, qui saturent de moins en moins la couche de surface du sol. Nous avons par contre recherché jusqu'à quelle tencur en eau maximum, ou plus exactement à partir de quelle teneur en air minimum, la production de $\mathrm{NO}_{3}-$ pouvait avoir lieu. Nous avons en effet supposé que l'air constituait le facteur critique de la nitrification (ainsi que lavaient fait Pilot \& PAtrick, 1972). Des auteurs ont pu relier le début de la production de $\mathrm{NO}_{3}-$ à un potentiel d'oxydo-réduction minimum, ce qui est logique; mais les valeurs citées sont parfois contradictoires, peut-être parce que la mesure de Eh n'est ni très sensible, ni très fidèle; de plus, Eh varie avec le temps, ce qui ne rend pas cette valeur d'utilisation très commode pour le problème qui nous intéresse. De toute façon, on peut penser que, pour un sol donné plus ou moins saturé d'eau, le potentiel d'oxydo-réduction dépenr surtout de la teneur en air.

Nous avons ainsi engorgé jusqu'en surface un poids donné de notre horizon $A_{1}$ dans de petits récipients décrits précédemment (dans cette expérience, le sol occupe une hauteur de 4 à $5 \mathrm{~cm}$ ). Ces récipients ont été mis en incubation pendant 24 jours, afin de produire une assez grande quantité de $\mathrm{NH}_{4}{ }^{+}$, matière première de la nitrification future; ils ont été remis en incubation après ressuyage, puis nous les avons retiré un à un, à une périodicité allant de un à quatre jours. A chaque date de prélèvement, nous avons mesuré, pour les récipients concernés, le poids d'azote nitrique ainsi que le volume occupé par le sol, et son humidité; on en déduit le volume occupé par l'air (fig. 3). 


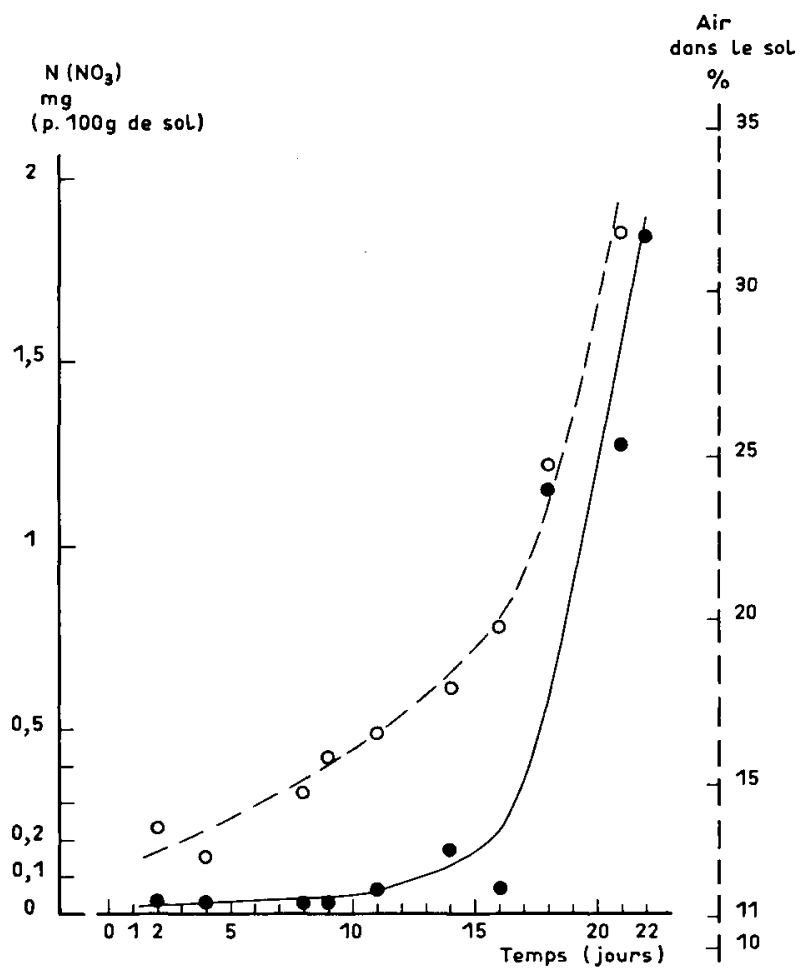

FIG. 3

Evolution comparée de la teneur en air du sol et de l'azote nitrique cumulé formé à partir du ressuyage $\left(a ̀{ }^{\circ} 7^{\circ} \mathrm{C}\right)$

Compared evolution of soil air content and cumulated nitric nitrogen produced since water-table removal $\left(\right.$ at $\left.27^{\circ} \mathrm{C}\right)$

On constate que :

- lallure générale des deux courbes $\left(\mathrm{NO}_{3}{ }^{-}\right.$et air en fonction du temps) est assez semblable;

- une production de $\mathrm{NO}_{3}{ }^{-}$est décelée pour la première fois au $11^{\mathrm{e}}$ jour. L'allure de la courbe $\mathrm{NO}_{3}^{-}$cumulé en fonction du temps est alors linéaire pendant quelques jours, cette droite débutant pour une teneur en air de 16 p. 100. On suppose donc que la nitrification commence au voisinage de cette dernière valeur. Il s'agit bien sûr de nitrification nette, c'est-à-dire de $\mathrm{NO}_{3}$ - formé moins $\mathrm{NO}_{3}$ - réorganisé, et moins $\mathrm{NO}_{3}{ }^{-}$réduit, principalement $\mathrm{NH}_{4}^{+}, \mathrm{N}_{2}, \mathrm{~N}_{22} \mathrm{O}$ (ces deux dernières formes entraînent une dénitrification, c'est-à-dire une perte par volatilisation); il y a probablement formation d'azote nitrique pour des teneurs en air inférieures à 16 p. 100, mais il est alors rapidement transformé ;

- le taux de la nitrification nette reste à peu près le même pour des teneurs en air comprises entre 16 et 19 p. 100 : il est faible ; 
- le taux de nitrification augmente considérablement à partir de 20 p. 100 d'air. L'optimum de teneur en air pour la nitrification débute probablement non loin de cette valeur.

En fait, le desséchement n'étant sans doute pas entièrement uniforme sur toute la hauteur du sol (4 à $5 \mathrm{~cm}$ ), les chiffres cités de la tencur en air, en particulier celui pour lequel débute la nitrification, sont peut-être un peu sous-estimés.

De plus, la liaison déduite de la figure 3 entre début de nitrification et un certain taux d'aération est en toute rigueur critiquable. En cffet, les valeurs de la teneur en air du sol évoluent dans le temps dans eette expérience; il y a ainsi probablement un eertain décalage entre la teneur en air permettant le démarrage de la nitrification et celle qui correspond à la présence dans le sol des premières quantités décelables de nitrates. On peut cependant penser que ce décalage est faible, car $\mathrm{NH}_{4}{ }^{+}$, substrat de la nitrification, est présent en assez grandes quantités dans le milieu, et d'autre part la méthode de dosage de $\mathrm{NO}_{3}^{-}$est très sensible. Cette remarque confirme néanmoins que les valeurs de teneur en air citées ci-dessus ne sont qu'approximatives.

La signification du taux de teneur en air qui correspond au début de la nitrification peut également être discutée. Cette valeur peut correspondre : fiantes,

- soit à un seuil d’aération nécessaire, dans tous les cas, aux bactéries nitri-

- soit à une levée d'inhibition de la nitrification; celle-ci débuterait lors de la disparition de produits inhibiteurs formés et accumulés durant l'engorgement du sol.

A l'encontre de cette seconde hypothèse, on peut d'une part supposer que le ressuyage élimine au moins en grande partie les produits inhibiteurs solubles; daautre part, comme nous le préciserons plus loin (3.4), le temps nécessaire à une reprise de la nitrification à la suite du ressuyage du sol engorgé est à peu près le même après différentes durées d'engorgement : cotte reprise serait done assez indépendante de la quantité de produits inhibiteurs éventuellement formés. On ne peut cependant retenir de façon définitive la $1^{\text {re }}$ hypothèse ; la valeur (approximative) de 16 p. 100 d'air ne constitue peut-être quiun indice, qui serait différent pour d’autres sols.

La nitrification débute donc dans ce sol pour une tencur en air peu éloignée de 16 p. 100. On peut supposer dans ces conditions que cest cette même teneur en air moyenne que contient. à proximité de la surface, ce même sol dans les vases de végétation lorsque la nappe est maintenue à $-6 \mathrm{~cm}$, c'est-à-dire à la profondeur pour laquelle débute l'assimilation de laazote par les plants d'Epicéa.

Nous avons done mesuré dans ces vases la teneur en air moyenne de la couche -1 à $-3 \mathrm{~cm}$, que l'on peut sans doute comparer valablement à celle des 4 ou $5 \mathrm{~cm}$ de sol des récipients qui avaient été placés en incubation. Nous avons utilisé pour cela des vases dépourvus de plants, dans lesquels nous avons nıcsuré l'humidité de la couche -1 à $-3 \mathrm{~cm}$, ainsi que sa densité apparente (à l'aide de petits cylindres). Nous aboutissons ainsi à une porosité à l'air (teneur en air en p. cent du volume de sol) de 16 p. 100.

Notre hypothèse est done confirméc : lorsque la nappe est proche de la surface, il n'y a pas d'azote assimilable (NO: ${ }^{-}$) disponible. De lazote commence à être 
assimilable par les jeunes plants d'Epicéa quand la nappe atteint une profondeur permettant le début de la nitrification en surface du sol. Il est probable que cet $\mathrm{NO}_{3}{ }^{-}$ est alors rapidement réduit s'il migre plus profondément dans le sol; mais l'une des adaptations des jeunes épicéas en sol engorgé consiste en la formation de racines prenant naissance peu en dessous du collet et dont la direction est subhorizontale (Levy, 1981) : il est vraisemblable que ce sont elles qui absorbent alors préférentiellement $\mathrm{NO}_{3}{ }^{-}$. D'ailleurs, même si de l'azote nitrique était disponible dans le cas d'un engorgement superficiel, les plants éprouveraient sans doute de grandes difficultés pour l'absorber, puis assimiler $\mathbf{N}$, dès que l'hypoxie priverait les cellules de l'énergie nécessaire à ces opérations (bien que $\mathrm{NO}_{3}$ puisse en partie compenser le déficit en oxygène).

\section{- Cas d'une nappe plus profonde}

On peut supposer que la quantité de $\mathrm{NO}_{3}-$ produit n'est pas suffisante tant que la teneur en air d'une grande partie de l'horizon $A_{1}$ n'atteint pas $16 \%$ ou même plus, c'est-à-dire tant que la nappe n'est pas suffisamment profonde. Mais on constate que l'assimilation de lazote est encore inférieure à celle du témoin lorsque la nappe est située à $-12 \mathrm{~cm}$ ou même plus profondément. On peut supposer qu'une partie des nitrates subit alors une réduction avant d'avoir pu être absorbée par les racines, parce qu'elle atteint, en migrant, des zones à potentiel d'oxydo-réduction faible : soit les quelques centimètres de sol situés directement au-dessus de la nappe, soit même, plus superficiellement, des «sites» plus humides que la moyenne du sol situé au même niveau (ce dernier phénomène se produit même dans des sols bien aérés : Dommergues et Mangenot, 1970).

Le développement du système racinaire dans les zones les mieux aérées du sol a donc probablement une certaine importance. De fait, nous avons constaté une meilleure assimilation relative de lazote lorsque la densité des racines était plus élevée, pour un même niveau de nappe : par exemple, sur la figure 1, $2^{\circ}$ date d'analyse de $\mathrm{N}$ par rapport à la $1^{\text {r". }}$.

\section{4. - Influence de l'ngorgement du sol sur la nutrition azotée de l'Epicéa au cours d'une saison de végétation en forêt}

En forêt, sur les sols à forte hydromorphie temporaire de l'Est de la France, la nappe ne demeure que rarement à proximité de la surface du sol aussi longtemps, du moins sans interruption, que dans nos essais de serre (par exemple, pour l'expérience de la figure 1 a, plus de 80 jours consécutifs), et cela à une période dactivité racinaire. On pourrait donc penser que la carence azotée provoquée par l'engorgement y est moins importante.

Or, on constate qu'en serre la tencur foliaire en azote, pour les plants qui avaient subi un engorgement de printemps suivi d'un maintien du sol à la capacité au champ, est à l'automne voisine de celle des plants non engorgés (il $y$ a donc eu «rattrapage » au cours de l'été) ; en forêt au contraire, une carence en azote apparaît à l'automne des années dont le printemps avait été humide (LEvy, 1978). Comment peut-on expliquer ce paradoxe? 
On pourrait penser que la nitrification in situ est toujours faible dans certaines des stations concernées, en raison du feutrage de champignons qui recouvre très souvent lhorizon $A_{1}$ sous peuplement forestier, ainsi que des lessivats de litière et éventuellement de mousses (Dommergues \& MANgenot, 1970). L'azote ne pourrait être assimilé qu'en sol bien ressuyé, car seule la forme $\mathrm{NH}_{4} \dashv$ serait disponible, ce qui expliquerait la carence azotée d'automne. Mais ce phénomène, s'il existe, n'est pas général, car nous avons constaté que la carence d'automne en azote apparaît dans des stations très variées, dont certaines sont dépourvues de feutrage et de litière, par exemple de jeunes plantations d'Epicéa en sol venant d'être labouré.

On pourrait également penser que les conditions climatiques régnant au cours de l'été sont souvent moins favorables à la minéralisation et labsorption de l'azote en forêt qu'en serre. Mais nos observations semblent indiquer que la carence d'automne apparaît souvent indépendamment du climat estival. Ce phénomène nést done pas général, mais il se produit probablement parfois: nous avons ainsi constaté une carence azotée d’automne faisant suite à une sécheresse estivale dans certains peuplements où les mauvaises propriétés physiques du sol limitaient sans doute l'enracinement ; cette carence ne faisait cependant pas suite à un important engorgement au cours du printemps de l'annéc considérée (Levy, 1978).

Un examen plus attentif du phénomène de la nitrification nous permet d'entrevoir un certain nombre d'autres explications :

\section{1) Tassement du sol}

L'horizon $A_{1}$ est en général plus tassé sous peuplement que dans nos vases de végétation, en serre. Alors que sa densité apparente variait de 0,60 (absence de nappe) à 0,79 (nappe à $-6 \mathrm{~cm}$ ) dans ce dernier cas, elle atteint des valeurs moyennes de 0,90 en forêt (il s'agit, rappelons-le, d'un humus limoneux, très fréquent dans les sols à hydromorphie temporaire). La nitrification est donc moins favorable en forêt, en sol engorgé; ainsi le début de formation de $\mathrm{NO}_{3}^{--}$exige sans doute une nappe plus profonde qu'en serre, bien que la température moins élevée en forêt constituc probablement un facteur favorable, du moins indircetement par l'intermédiaire du potentiel d'oxydo-réduction (car une température basse a un effet direct négatif sur la nitrification).

\section{2) Durće nécessaire à la reprise de la nitrification}

Nous avons étudié, par incubations, l'évolution de la tencur en $\mathrm{NO}_{3}^{-}$dans l'horizon $A_{1}$, à la suite d'un engorgement en surface et à $-4 \mathrm{~cm}$ pendant des durées variables ( 7,25 et 52 jours), suivi d'un ressuyage et d'un maintien du sol à la capacité au champ, également pendant des durées variables, et cela dans chacun des cas. La figure 4 représente les résultats de cette expérience pour un engorgement de 52 jours; ils sont dans l'ensemble semblables pour les deux autres durées.

Nous avons vu qu'à la fin d'un engorgement de surface, la nitrification (donc l'assimilation de l'azote par l'Epicéa) ne peut reprendre que lorsque le sol est suffisamment ressuyé (teneur en air voisine de 16 p. 100, pour l'horizon $A_{1}$ utilisé). Dans cette expérience, la nitrification ne reprend ainsi d'une façon sensible qu'entre 18 et 36 jours après le ressuyage; d'après l'allure de la courbe, ce délai est probablement de l'ordre de 20 à 25 jours, et cela indépendamment de la durée de l'engorgement 
(même s'il ne dure qu'un seul jour, nous l’avons constaté dans une autre expérience). Lorsque la nappe était située à $-4 \mathrm{~cm}$ et non plus en surface, la nitrification reprend plus tôt, entre 0 et 18 jours après le ressuyage : le seuil d’aération est alors attcint plus rapidement.

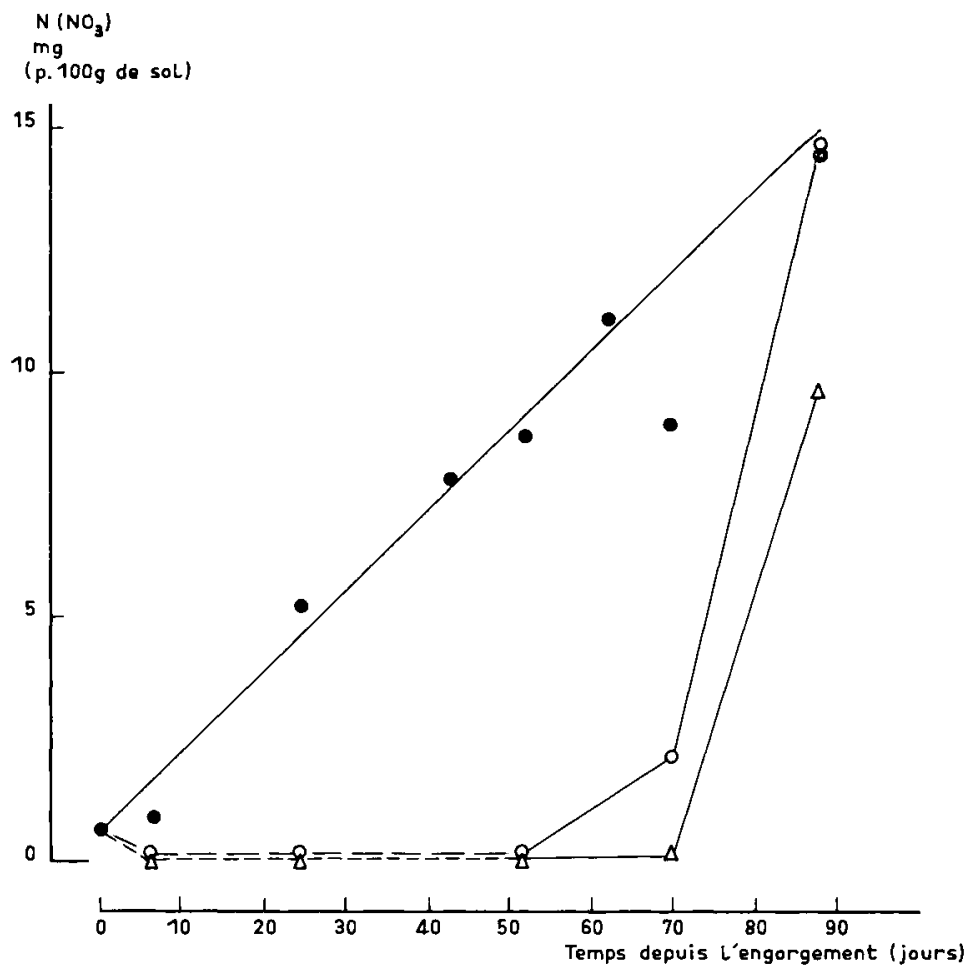

Fig. 4

Evolution de la teneur du sol en azote nitrique pour diverses conditions hydriques $\left(\dot{a} 27^{\circ} \mathrm{C}\right)$

Evolution of soil nitric nitrogen content for different soil water conditions (at $27^{\circ} \mathrm{C}$ )

Capacité au champ

Field capacity

- - Nappe à $-4 \mathrm{~cm}$

Water-table at $4 \mathrm{~cm}$ deep

..... Nappe en surface

Surface water logging

Ces «temps morts» (de durée sans doute variable en forêt) se produisent probablement à plusieurs reprises au cours de la même année dans certaines stations, selon la fluctuation des nappes. 


\section{3) Transformations d'azote}

a) Lors de l'engorgement : on sait que l'engorgement d'un sol provoque une réduction presque totale de $\mathrm{NO}_{3}$ dans la zone ennoyée. La figure 4 montre qu'il en est pratiquement de même dans les quelques centimètres qui sont situés directement au-dessus de la nappe.

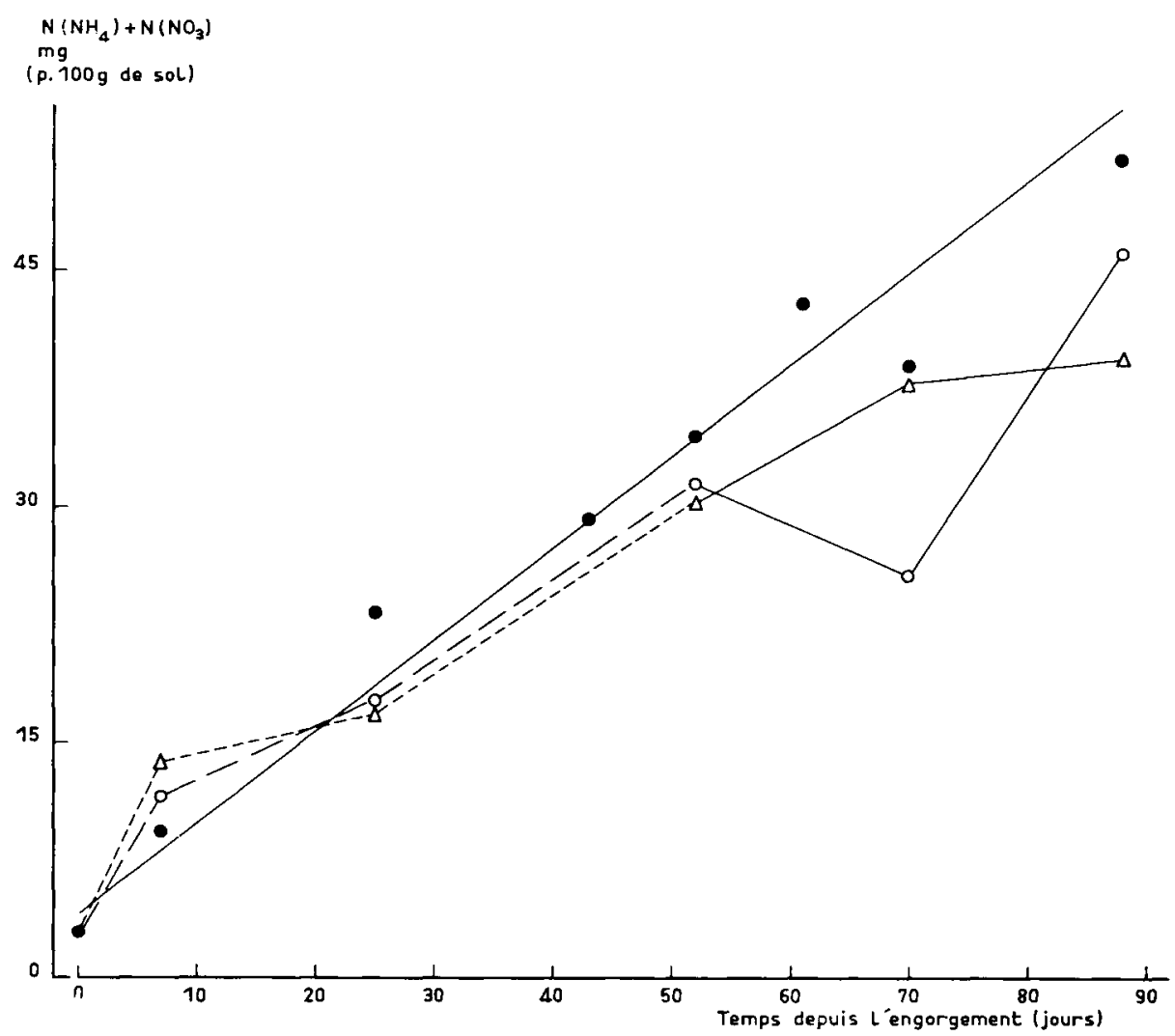

FIG. 5

Evolution de la teneur du sol en azote ammoniacal + nitrique pour diverses conditions hydriques (à $27^{\circ} \mathrm{C}$ )

Evolution of soil $\mathrm{NO}_{3}-+\mathrm{NH}_{4}+$ nitrogen for different soil water conditions (at $27^{\circ} \mathrm{C}$ )

Capacité au champ

Field capacity

- - Nappe à $-4 \mathrm{~cm}$

Water-table at $4 \mathrm{~cm}$ deep

...... Nappe en surface

Surface water logging 
b) Ultérieurement : l'azote nitrique que l'on mesure peut être en réalité la résultante de deux processus : nitrification moins réduction ou/et réorganisation. Aussi avons-nous tenté de voir si des pertes d'azote minéral se produisent à d'autres moments que lors de l'engorgement. Dans ce but, nous avons tracé, pour l'expérience correspondant à la figure 4, l'évolution de la teneur du sol en $\mathrm{NO}_{3}-+\mathrm{NH}_{4}{ }^{+}$(fig. 5). On peut constater que, pour le sol maintenu à la capacité au champ depuis l'origine, cette valeur augmente toujours à peu près uniformément avec le temps (aussi avonsnous tracé, pour ce régime hydrique, une droite moyenne). Il en est de même durant la période d'engorgement avec une nappe située en surface ou à $-4 \mathrm{~cm}$; puis la pente de la droite diminue sensiblement pendant une certaine période. Ce dernier phénomène apparaît entre 0 et 18 jours après le ressuyage lorsque la nappe était située à $-4 \mathrm{~cm}$, entre 18 et 36 jours après le ressuyage quand elle était superficielle ; la comparaison avec la figure 4 montre que ce phénomène intervient, dans chacun des deux cas, durant la période de reprise de la nitrification. Il correspond à une perte d'azote minéral; deux types de transformations sont ainsi susccptibles de se produire, simultanément ou non : dénitrification d'une partie au moins de $\mathrm{NO}_{3}-$ qui vient de se former (l'azote correspondant est définitivement perdu) et réorganisation (cet azote n'est perdu que provisoirement pour la végétation).

Ces transformations et pertes d'azote à l'engorgement puis lors de la reprise de la nitrification interviennent sans doute à plusieurs reprises au cours d'une même année, en forêt, car la nappe fluctue sans arrêt. Ainsi Burschel et al. (1977) ont constaté, sous un peuplement de Pin fertilisé, une très forte perte d'azote de l'écosystème; cette perte est apparemment provoquée surtout par dénitrification à la suite de l'alternance stagnation de l'eau-dessèchement en surface du sol.

4) Pertes par lessivage: la figure 4 montre que lorsque la nitrification peut reprendre après une longue période d'engorgement du sol, elle a une allure presque "explosive »: sa vitesse est très élevée (car sa matière première $\mathrm{NH}_{4}+$ est abondante) ; il est plausible que des pertes d'azote puissent alors se produire par lessivage des nitrates, au moins si des pluies importantes interviennent. D'ailleurs, les fluctuations de la nappe provoquent sans doute aussi parfois le lessivage d'une partie de lazote ammoniacal, lorsqu'il s'est accumulé en quantité telle qu'il ne peut être entièrement retenu par le complexe absorbant du sol.

\section{IV. - Conclusion}

Des études expérimentales menées les unes en vases de végétation sur substrat dépourvu de matière organique, les autres en incubation sur horizon $A_{1}$ limoneux, nous ont permis de proposer une explication à la soudaine et totale carence azotée affectant de jeunes plants d'Epicéa en présence d'une nappe affleurant à proximité de la surface du sol.

Il est ainsi apparu que l'azote ammoniacal n'était jamais assimilé en présence d'une nappe affleurant entre la surface et au moins $-17 \mathrm{~cm}$, alors que les conditions nécessaires à l'assimilation de l'azote nitrique étaient beaucoup moins sévères. La nutrition de l'Epicéa dans ce cas ne peut donc s'effectuer qu'à partir de $\mathrm{NO}_{3}{ }^{-}$, mais 
encore faut-il que celui-ci soit disponible. Or, la nitrification ne peut se produire que si le sol n'est pas trop humide : elle débute lorsque le sol utilisé contient environ $16 \%$ d'air en volume. Cela explique que dans nos conditions expérimentales, les plants d'Epicéa n'assimilent l'azote provenant d'une minćralisation de la matière organique que lorsque la nappe est située à environ $-6 \mathrm{~cm}$ (profondeur pour laquelle le sol contient 16 p. 100 d'air près de sa surface) ou en dessous.

D'autre part, de faibles quantités d'azote nitrique épandues sur le sol (ainsi d'ailleurs que le peu de $\mathrm{NO}_{3}{ }^{-}$qui pouvait préexister) sont totalement réduits, donc non disponibles pour les plants, lorsque la nappe est relativement superficielle.

Les phénomènes de nitrification et réduction de $\mathrm{NO}_{3}^{-}$expliquent bien l'allure des courbes représentant la teneur en azote des aiguilles en fonction de la profondeur de la nappe.

Nous avons d'autre part tenté de trouver une explication au fait qu'en forêt, contrairement à ce que l'on constate en serre, on décèle encore à l'automne des années dont le printemps avait été humide une carence en azote dans les fcuilles d'Epicéa sur sols à forte hydromorphie temporaire.

Il apparaît qu'au cours de ces années-là, la période cumulée pendant laquelle il n'y a pas d'azote assimilable disponible est certainement longue, surtout si l'engorgement remonte parfois jusqu'en surface, en raison des conditions hydriques nécessaires à la reprise de la nitrification et de celles indispensables à l'assimilabilité de l'azote ammoniacal.

De plus, certaines des fluctuations continuelles de la nappe entraînent des pertes répétées d'azote assimilable lors des engorgements (s'il existe alors de l'azote nitrique) puis au moment de la reprise de la nitrification consécutive au ressuyage.

Enfin, il y a probablement un lessivage assez important d'azote minéral, soit (un peu) sous forme ammoniacale par entraînement par la nappe de $\mathrm{NH}_{4}+$ qui s'est accumulé pendant l'engorgement, soit surtout sous forme nitrique après la nitrification intense qui fait suite à une longue période d'engorgement.

Reçu pour publication en janvier 1980.

\author{
Summary \\ Nitrogen nutrition of Norway Spruce in water-logged soil : \\ experimental study
}

In experimental conditions, young plants began assimilating nitrogen only when water-table was about at a minimum depth of $6 \mathrm{~cm}$. For deeper water levels, the deeper was the water-table, the better was nitrogen foliar content.

Nitrogen nutrition of Spruce in water-logged soil depends on the presence of nitric nitrogen. Indeed, on one hand, nitrogen brought in $\mathrm{NH}_{4}+$ form was not assimilated at all even when the water-table was deep, contrary to $\mathrm{NO}_{3}-$ form. $\mathrm{NH}_{4}+$ is only absorbed when there is no water-table. On the other hand, the minimum air content which enabled nitrification was obtained, near soil surface, when the water-table was $6 \mathrm{~cm}$ deep : nitrogen assimilation by the plants began for this same level. 
Incubation experiments showed that, in forest, several phenomena can prolong the period of Spruce nitrogen deficiency. Thus, every time the water-table is near soil surface, nitrification only begins again a long time after water-table removal; moreover, a loss of mineral nitrogen takes place then; if soil was water-logged for a long time, a great quantity of $\mathrm{NO}_{3}-$ is produced during a short period of time : losses of nitrates by leaching may therefore occur.

\section{Références bibliographiques}

Burschel P., Eder R., Kantarci D., Rehfuess K.E., 1977. Effects of different soil cultivation practices on the growth, phytomass accumulation and nutrient balance in young pine forest ecosystems (Pinus sylv. L.). Forstw. Zentrall., 96 (6), 321-338.

Dommergues Y., Mangenot F., 1970. Ecologie microbienne du sol. Masson et $\mathrm{C}^{\text {le }}$, éditeurs, Paris, $796 \mathrm{p}$.

Heller R., 1969. Biologie végétale. II. Nutrition et métabolisme. Masson et $\mathrm{C}^{\text {te }}$, éditeurs, Paris, $578 \mathrm{p}$.

Levy G., 1971. Influence de l'engorgement de printemps et de la sécheresse d'été sur le comportement de jeunes plants d'Epicéa. Ann. Sci. for., 28 (4), 403-423.

LeVY G., 1978. Nutrition et production de l'Epicéa commun adulte sur sols hydromorphes en Lorraine : liaisons avec les caractéristiques stationnelles. Ann. Sci. for., 35 (1), 33-53.

Levy G., 1981. Comportement de jeunes plants d'Epicéa commun en sol à engorgement temporaire de surface : influence de divers facteurs du milieu. Ann. Sci. for., 38 (1).

Pinot L., Patrick W.H., 1972. Nitrate reduction in soils : effect of soil moisture tension. Soil Sci., 114 (4), 312-316. 